\title{
PRESENÇA DE HPAS EM AMOSTRAS DE AR ATMOSFÉRICO ORIUNDO DE AMBIENTES FECHADOS - UMA BREVE REVISÃO
}

Marinice Santiago dos Santos Acácio ${ }^{1 *}$; Jeancarlo Pereira dos Anjos ${ }^{1}$; Bruna Aparecida Souza Machado1 - *marinicesantiago@yahoo.com.br

${ }^{1}$ Centro Universitário SENAI CIMATEC - Av. Orlando Gomes, 1845 - Piatã, 41650010, Salvador - BA

Resumo: Este trabalho teve como objetivo realizar uma breve revisão acerca de métodos utilizados para a determinação de HPAs em amostras de ar atmosférico oriundo de ambientes fechados. Os artigos foram selecionados, após consulta em diferentes bases de dados e avaliados de acordo com a aderência ao tema. Membranas de quartzo, filtros de microfibra de vidro e filtro de PTFE têm sido usados durante a etapa de coleta das amostras. No processo de extração dos analitos, diclorometano e mistura de solventes têm sido empregados. Os analitos são quantificados usando as técnicas de GC-MS ou HPLC-FLD. A quantificação de HPAs em ar ambiente evidencia a eficácia dos métodos apresentados, tornando-se importante a implementação de métodos de rotina para o monitoramento destas substâncias em ambientes fechados.

Palavras-Chave: ar atmosférico; HPAs; monitoramento, poluentes.

\section{PRESENCE OF HPAS IN ATMOSPHERIC AIR SAMPLES FROM INDOORS ENVIRONMENTS - A BRIEF REVIEW}

\begin{abstract}
This work aimed to perform a brief review about the methods used for the determination of PAHs in atmospheric air samples from indoor environments. The articles were selected after consultation in different databases and evaluated according to their adherence to the theme. Quartz membranes, glass microfiber filters and PTFE filters have been used during the sample collection step. In the process of the analytes extraction, dichloromethane and solvent mixing have been employed. Analytes are quantified using GC-MS or HPLC-FLD techniques. The quantification of $\mathrm{PAHs}$ in ambient air shows the effectiveness of the methods presented, making it important to implement routine methods for monitoring these substances indoors.
\end{abstract}

Keywords: atmospheric air; HPAs; monitoring; pollutants 


\section{INTRODUÇÃO}

A diminuição da qualidade de vida, cada vez mais afetada por fatores resultantes das diversas atividades humanas nos mais diferentes aspectos, vem sendo um assunto bastante discutido nos dias atuais. Doenças respiratórias, cardiovasculares e neurológicas têm sido constantemente associadas à poluição atmosférica, na qual a presença de poluentes vem atingindo níveis preocupantes [1].

O uso e a ocupação indevida do solo, o processo de combustão nos veículos automotores, as atividades industriais nos centros urbanos, os processos erosivos e a expansão urbana sem planejamento são as principais fontes de poluentes e responsáveis pela contaminação do ar atmosférico [2]. Diferentes estudos mostram que estes agentes são nocivos à saúde da população exposta, mesmo quando presentes em baixa concentração [3].

Buscando minimizar os efeitos causados pelos mais variados tipos de poluentes na atmosfera, diversos países passaram a formular seus padrões para monitorar a qualidade do ar, estabelecendo limites de tolerância para alguns parâmetros e classes de compostos, a fim de reduzir os efeitos negativos destas substâncias na saúde da população [3].

O monóxido de carbono (CO), materiais particulados (MP), óxido de nitrogênio $\left(\mathrm{NO}_{\mathrm{x}}\right)$, óxido de enxofre $\left(\mathrm{SO}_{\mathrm{x}}\right)$ e hidrocarbonetos $(\mathrm{HC})$ são alguns dos poluentes presentes no ar atmosférico e cujo monitoramento requer uma maior atenção por parte do poder público, tendo em vista os efeitos nocivos para a saúde da população exposta [4].

Dentre os hidrocarbonetos, pode-se destacar os HPAs (Hidrocarbonetos Policíclicos Aromáticos), que são subprodutos formados durante a combustão incompleta de matéria orgânica, e cujas características cancerígenas já foram devidamente comprovadas para algumas espécies químicas [5].

Devido às características carcinogênicas destas substâncias, 16 compostos da classe dos HPAs (Naftaleno, Acenafteno, Acenaftileno, Antraceno, Fluoreno, Fenantreno, Fluoranteno, Pireno, Benzo(a)antreceno, Criseno, Benzo(b)fluoranteno, Benzo(k)fluoranteno, Dibenzo(a,h)antraceno, Benzo(a)pireno, Indeno(1,2,3cd)pireno, Benzo(g,h,i)perileno), foram considerados prioritários e selecionados, pela Agência de Proteção Ambiental Americana (USEPA), para monitoramento em diferentes compartimentos ambientais, incluindo no ar atmosférico [6]. A Figura 1 mostra a estrutura destes compostos. 
Figura 1 - 16 HPAs prioritários de acordo com a EPA [7]

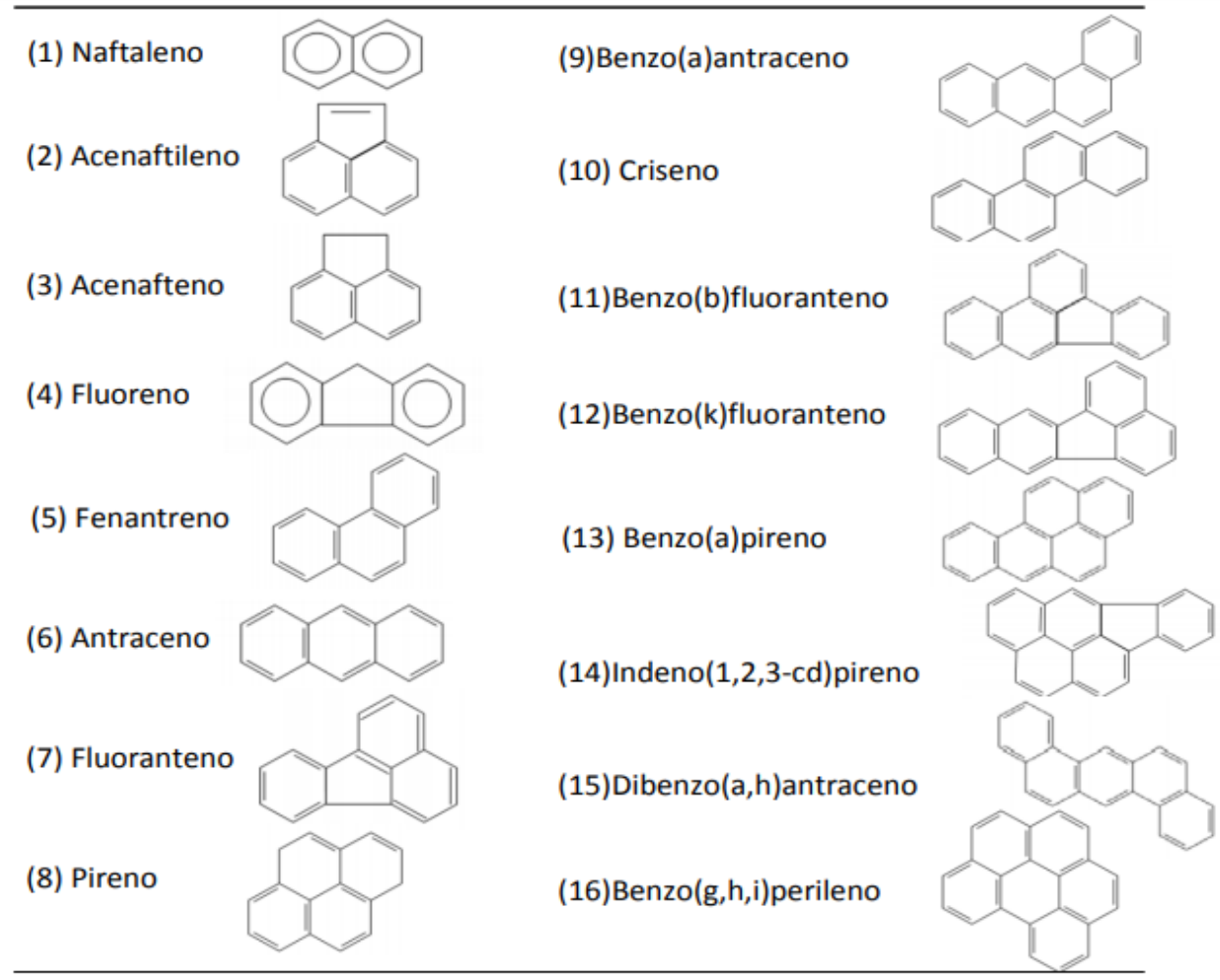

No Brasil, os padrões de qualidade do ar são regulamentados por meio da Resolução do Conselho Nacional de Meio Ambiente (CONAMA) № 491, de 19 de novembro de 2018, que estabelece os limites de tolerância para alguns poluentes [8].

Embora se tenha conhecimento sobre a importância do gerenciamento da qualidade do ar, no Brasil, muitos agentes poluentes ainda não são monitorados e controlados pela legislação, a exemplo dos HPAs que são extremante nocivos à saúde humana. Nesse sentido, a implantação de programas de monitoramento, como o que preconiza a Agência de Proteção Ambiental dos Estados Unidos (USEPA), torna-se de extrema urgência para que se tenha uma melhoria da qualidade do ar atmosférico o qual a população está exposta [9].

Visando contribuir no processo de monitoramento de agentes poluentes no ar atmosférico, este trabalho teve como objetivo realizar uma breve revisão acerca de métodos utilizados para a determinação de HPAs em amostras de ar atmosférico provenientes de ambientes fechados a fim de avaliar os riscos de exposição a estas substâncias poluentes.

\section{METODOLOGIA}

Foi realizada uma busca por artigos científicos publicados entre os anos de 2014 a 2019, nas bases de dados das plataformas Google Acadêmico, Scielo e ScienceDirect. Os termos ar atmosférico, HPAs, monitoramento, poluentes, 
atmospheric air, monitoring e pollutants, foram usados como palavras-chaves para a pesquisa. Para restringir a pesquisa foi feito o cruzamento dos termos usando 0 operador AND. Assim, 39 artigos foram selecionados. Após a leitura dos títulos e resumos, 14 artigos foram incluídos nesta revisão considerando a aderência ao tema e observando os seguintes pontos: (i) tipo de ambiente de coleta de amostras, (ii) tipos de amostradores utilizados na coleta das amostras, (iii) solventes utilizados para o preparo de amostras e (iv) técnicas utilizadas para identificar e quantificar os HPAs. Foram excluídos 25 títulos por não estarem relacionados ao objetivo da pesquisa.

\section{RESULTADOS E DISCUSSÃO}

Os HPAs são compostos orgânicos, da classe dos hidrocarbonetos, que contém em sua estrutura dois ou mais anéis aromáticos. São componentes de misturas complexas, podendo ser encontrados em plantas, solos, sedimentos, águas continentais e marinhas e no ar atmosférico. Além disso, são substâncias semivoláteis sendo, portanto, encontrados na atmosfera, tanto na fase gasosa quanto na fase de partículas [10].

A exposição dos seres humanos aos HPAs pode ocorrer por diversas formas tais como, ingestão de alimentos contaminados, fumaça de cigarros, inalação de fumaças de lareiras abertas, pela respiração em local com ar ambiente contaminado, entre outras [11]. A inalação de material particulado atmosférico contendo HPAs, entretanto, é considerada a principal forma de exposição à estas substâncias [12].

Estudos têm evidenciado que o ar interior oriundos de ambientes fechados e semifechados também estão propensos a concentração destes contaminantes.

Romagnoli et al. (2014) [13], monitoraram dezoito ambientes distintos "escolas, escritórios de ambientes não industriais e residências", com o objetivo de avaliar o nível de exposição e o impacto na saúde das pessoas expostas. Neste trabalho a coleta do material particulado das amostras foi realizada usando membrana de filtro de quartzo pré-aquecida a $600^{\circ} \mathrm{C}$ por um período de $6 \mathrm{~h}$ e acondicionada em dessecador até o momento do uso. Uma mistura de solventes contendo acetona, hexano e tolueno na proporção (60:30:10), foi usada como como solvente extrator no processo de extração. A cromatografia gasosa acoplada a espectrometria de massa (GC-MS) foi a técnica usada para quantificar as amostras. 08 espécies de HPAs foram encontrados. As concentrações encontradas variaram de 0,1 a 1,6 ng/m ${ }^{3}$ para o benzo (a) pireno e de 1,8 a $8,4 \mathrm{ng} / \mathrm{m}^{3}$ para HPAs total. Os autores observaram que a qualidade do ar em ambientes de vivência e de trabalho são afetados por poluentes cancerígenos, devido aos altos índices de HPAs encontrados.

No estudo realizado por Vukovic et al. (2014) [14], foi analisado o ar atmosférico interno presente em quatro garagens de estacionamento semifechadas na cidade Belgrado, na Sérvia. O objetivo deste trabalho foi avaliar a presença de HPAs e outros poluentes nestes ambientes. O material particulado foi coletado usando membrana de quartzo previamente acondicionada por 48 horas. Diclorometano foi usado como solvente na etapa de extração. A cromatografia gasosa acoplada a espectrometria de massa (GC-MS) foi a técnica utilizada para quantificar as amostras. Neste estudo os 
16 HPAs prioritários foram quantificados. As concentrações de HPAs total encontradas variaram de 13 a $42 \mathrm{ng} / \mathrm{m}^{3}$. Tais resultados evidenciam a necessidade do monitoramento da qualidade do ar nestes ambientes.

Sabino et al. (2015) [15], analisaram o ar interno de uma estação de ônibus semifechada com o objetivo de verificar a presença de HPA e Nitro-HPA, associado a fração fina de MP proveniente da combustão de mistura de diesel/biodiesel. A amostragem foi feita usando filtro de PTFE previamente acondicionado. Após a coleta as membranas foram armazenadas em folhas de alumínio e refrigeradas a $4^{\circ} \mathrm{C}$ até 0 momento da extração. No processo de extração o diclorometano foi usado como solvente extrator. A técnica de cromatografia líquida de alta eficiência com detector de fluorescência (HPLC-FLD) foi usada na quantificação dos HPAs. 9 dos 16 HPAs prioritários foram identificados. As concentrações totais de HPAs nas frações de $\mathrm{PM}_{2,5}$ e $\mathrm{PM}_{10}$ foram 178 e $118 \mathrm{ng} / \mathrm{m}^{3}$ respectivamente. Resultado que mostra que o monitoramento da qualidade do ar em ambiente fechado e semifechado é uma necessidade aparente.

No trabalho realizado por Singh et al. (2016) [16], foi investigada a qualidade do ar interno de uma cozinha, cuja fonte de poluentes inclui o material particulado oriundo da queima do óleo de cozinha, comumente conhecido como "vapores de cozinha". O objetivo deste foi medir a qualidade do ar interno da cozinha, estimar e avaliar os riscos dos HPAs. Membranas de microfibra de vidro foram empregadas como amostradores na coleta do material particulado. As amostras foram extraídas com auxílio de um ultrassom usando como extrator uma mistura de solventes, composta por acetona e n-hexano (1:1). Posteriormente foi usado filtros de PTFE para filtrar as amostras antes da injeção. A quantificação dos analitos foi feita usando a cromatografia liquida de ultra eficiência com detector de fotodiodos PDA (UHPLCPDA). Os 16 HPAs prioritários foram quantificados. A soma das médias das concentrações foi de $51,13 \mu \mathrm{g} / \mathrm{m}^{3}$. Tal resultado reitera a necessidade do monitoramento destes espaços.

Filtros de quartzo [13,14], filtros de PTFE [16] e membranas de microfibra de vidro [16], foram usados como amostradores para coleta do material particulado. No preparo das amostras, a técnica de extração por solvente foi usada em todos os trabalhos [13 14,15,16 ]. Acetona, hexano e tolueno misturados proporcionalmente em (60:30:10) [13], Diclorometano [14,15] e uma mistura de acetona e n-hexano na proporção 1:1[16], foram usados como solventes extratores na etapa de extração. (GC-MS) [13,14], (HPLC-FLD) [15], (UHPLC-DAD) [16], foram as técnicas cromatográficas aplicadas para a quantificação dos HPAs. O interior de "escolas, escritórios de ambientes não industriais e residências'[13], garagens[14], estação de ônibus[15] e cozinha [16], foram os ambientes monitorados para fim de verificar a presença de HPAs.

Diante do exposto, fica evidente que a presença de HPAs em ambientes fechados é um problema atual em diferentes setores da sociedade, despertando o interesse para o desenvolvimento de métodos mais rápidos e econômicos para a determinação rotineira da concentração destas espécies no ar atmosférico. 
Ainda com base nos resultados apresentados, os estudos apontaram uma preocupação no tocante a qualidade de vida, quando o assunto é contaminação por agentes poluentes, particularmente os HPAs.

Em um número significativo de artigos selecionados [13,14,15,16], observouse ainda que as metodologias aplicadas para a determinação de HPAs em ar atmosférico em ambientes fechados foram realizadas de forma semelhantes. Identificou-se ainda que a extração com solventes orgânicos tem sido a técnica mais utilizada, sendo o diclorometano o solvente mais empregado. Dessa forma, como sugestão de melhoria contínua, torna-se relevante o desenvolvimento de estudos envolvendo o uso de novas metodologias de análise, ou otimização das já existentes.

Para a quantificação de analitos traços, como é caso dos HPAs, técnicas cromatográficas, GC-MS, HPLC-FLD e UHPLC-DAD foram aplicadas de forma eficaz. Estas técnicas, embora tenham altos custos, apresentam sensibilidade e seletividade adequadas para este tipo de análise.

\section{CONSIDERAÇÕS FINAIS}

Os resultados encontrados nos estudos avaliados nos ambientes fechados e semifechados, quanto a presença de HPAs apresentaram-se preocupantes, evidenciando a necessidade de monitoramento destes ambientes de modo a avaliar o nível de exposição humana a estes poluentes.

Observou-se que o uso de filtros de quartzo, membranas de fibra de vidro e filtros de PTFE têm se mostrado adequado e eficiente para coleta de amostras de material particulados de ar atmosférico em ambientes fechados. Entretanto, a pesquisa por outros tipos de amostradores pode ser considerada uma possibilidade de melhoria no processo de amostragem.

O diclorometano, de modo geral, ainda é o solvente mais usado nos processos de extração por solvente. Nesse aspecto a realização de estudos envolvendo outros solventes é pertinente.

O desenvolvimento de metodologias com custos mais baixos poderá contribuir para o monitoramento da concentração de HPAs na atmosfera, uma vez que possibilitará a implementação de métodos de rotina em diferentes laboratórios, principalmente para a avaliação da presença destes poluentes em ambientes fechados, dada a toxicidade características destes compostos.

\section{REFERÊNCIAS}

1DE BRITO, P. H.; ARAÚJO, R. dos S.; MARINHO, G. Composição Química do Material Particulado Atmosférico: Uma Revisão de Literatura. HOLOS, v. 4, p. 62-74, 2018. 
${ }^{2}$ FARIA, F. do N.; BARBOSA, K. V.; RIBEIRO, O. P. Levantamento dos Processos Intervenientes na Concentração de Material Particulado no Município de Campos dos Goytacazes/RJ, Boletim do Observatório Ambiental Alberto Ribeiro Lamego, v.12 n.1, p. 57-88, jan. /jun. 2018

${ }^{3}$ DAPPER, S. N.; SPOHR, C.; ZANINI, R. R. Poluição do ar, como fator de risco para a saúde: uma revisão sistemática no estado de São Paulo. Estudos Avançados, v. 30, n. 86, p. 83-97, 2016.

${ }^{4}$ OLIVEIRA, A. P. de; MATOS, M. C. N. de; PEREIRA, B. B. Avaliação da Exposição Ambiental ao Monóxido de Carbono, Material Particulado e ao Ruído no Terminal Central de Transporte Coletivo de Uberlândia, Minas Gerais. J. Health Biol. Sci. (Online), v. 5, n. 1, p. 79-85, 2017.

${ }^{5}$ MONTEIRO, D. dos S; TAVARES, T. M.; OLIVA, S. T. Investigação da Presença de Hidrocarbonetos Aromáticos em Poços Freáticos ao Norte do Recôncavo da Bahia, Brasil. Revista Eletrônica de Gestão e Tecnologias Ambientais (GESTA), v. 4, n. 2 p. 129-140, 2016 - ISSN: 2317-563X

${ }^{6}$ EPA, U. S. Locating and estimating air emissions from sources of polycyclic organic matter. Research Triangle Park (NC): United States Environmental Protection Agency, 1998.

7 SANTOS, L. O. Estudo de HPA's, nitro-HPA's e quinonas em amostras ambientais. Tese (Doutorado em Ciências, Energia e Ambiente). Universidade Federal da Bahia (UFBA), Centro Interdisciplinar de Energia e Ambiente (CIEnAm), 2017.

${ }^{8}$ BRASIL. Conselho Nacional do Meio Ambiente (CONAMA). Resolução no:491, de 19 de novembro de 2018. Dispõe sobre Padrões de Qualidade do Ar, previstos no PRONAR. Diário Oficial da União, Brasília, DF, 21 de novembro de 2018,

${ }^{9}$ RIBEIRO, A. P. et al. Diagnóstico da Poluição Atmosférica em Regiões sem Redes Convencionais de Monitoramento da Qualidade do Ar: Estudo em uma Pequena Cidade do Paraná, Brasil. Interciencia, v. 42, n. 11, p. 767-773, 2017.

${ }^{10}$ HASSINE, S. B.; HAMMAMI, B.; AMEUR, B. W.; MEGDICHE, E. Y.; BARHOUMI, B.; DRISS, M.R. Particulate polycyclic aromatic hydrocarbons (PAH) in the atmosphere of Bizerte City, Tunisia. Bulletin of environmental contamination and toxicology, v. 93, n. 3, p. 375-382, 2014

${ }^{11}$ ABDEL-SHAFY, H. I.; MANSOUR, M. S. M. A review on polycyclic aromatic hydrocarbons: source, environmental impact, effect on human health and remediation.

Egyptian Journal of Petroleum, v. 25, n. 1, p. 107-123, 2016.

${ }^{12} \mathrm{ROCHA}$, S. M.; NISHIO, F. Y. Contaminação por Hidrocarbonetos Policíclicos Aromáticos (HPAs) em Mananciais: evidências de risco à saúde no Município de São Paulo. InterfacEHS, v. 11, n. 1, 2016.

${ }^{13}$ ROMAGNOLI, P.; BALDUCCIA, C.; PERILLIA, M.; GHERARDIB, M.; GORDIANIB, A.; GARIAZZOB; GATTOB , M.P.; CECINATOA , A. Indoor PAHs at schools, homes and offices in Rome, Italy. Atmospheric Environment, v. 92, p. 51-59, 2014.

${ }^{14}$ VUKOVIĆ, G.; UROŠEVIĆ, M. A.; RAZUMENIĆ, I.; KUZMANOSKI, M.; PERGAL, M.; ŠKRIVANJ, S.; POPOVIĆ, A. Air quality in urban parking garages (PM10, major 
and trace elements, PAHs): Instrumental measurements vs. active moss biomonitoring. Atmospheric Environment, v. 85, p. 31-40, 2014.

${ }^{15}$ SABINO, F. C.; PINTO, J. P.; PIRACELLI, V. P.; AMADOR, I. R.; MARTINS, L.D.; HOFFMANN, T.; SOLCI, C. HPA e Nitro-HPA em Ambiente Semifechado Impactado por Emissão da Combustão de Diesel/Biodiesel (B5). Orbital: The Electronic Journal of Chemistry, v. 7, n. 3, p. 245-252, 2015.

${ }^{16}$ SINGH, A.; NAIR, K.C.; KAMAL, R.; BIHARI,V.; GUPTA,M.K.; MUDIAM, M. K R.; SATYANARAYANA, G.N.V.; RAJ, A.; HAQ,I.; SHUKLA, N. K.; KHAN, A.H.; SRIVASTAVA, A.K. Assessing hazardous risks of indoor airborne polycyclic aromatic hydrocarbons in the kitchen and its association with lung functions and urinary PAH metabolites in kitchen workers. Clinica Chimica Acta, v. 452, p. 204-213, 2016. 